Journal of Applied Mathematics and Stochastic Analysis, 16:3 (2003), 243-248.

Printed in the USA (C)2003 by North Atlantic Science Publishing Company

\title{
COMMON FIXED POINT THEOREMS FOR A PAIR OF COUNTABLY CONDENSING MAPPINGS IN ORDERED BANACH SPACES
}

\author{
B.C. DHAGE \\ Kasubai, Gurukul Colony \\ Almedpur 413515, Maharashtra, India \\ DONAL O'REGAN \\ National University of Ireland \\ Department of Mathematics \\ Galway, Ireland \\ RAVI P. AGARWAL \\ Florida Institute of Technology \\ Department of Mathematical Sciences \\ Melbourne, FL 32901 USA
}

(Received January, 2003; Revised June, 2003)

In this paper some common fixed point theorems for a pair of multivalued weakly isotone mappings on an ordered Banach space are proved.

Keywords. Common Fixed Point, Multivalued, Weakly Isotone, Ordered Banach Space.

AMS (MOS) subject classification. 47A10.

\section{Introduction}

Let $X$ be a Banach space with norm $\|$.$\| . A nonempty closed subset K$ of $X$ is called a cone if

(i) $K+K \subseteq K$,

(ii) $\lambda K \subseteq K$ for all $\lambda>0$ and

(iii) $(-K) \cap K=\{0\}$ where 0 is the zero element of $X$.

We define an order relation $\leq$ in $X$ with the help of the cone $K$ as follows: for $x, y \in X$ then $x \leq y$ iff $y-x \in K$. By an ordered Banach space $X$ we mean the 
Banach space $X$ equipped with a partial ordering $\leq$ induced by $K$. Let $z, w \in X$ be such that $z \leq w$. Then by an order interval $[z, w]$ we mean a set in $X$ defined by

$$
[z, w]=\{x \in X: z \leq x \leq w\} .
$$

A cone $K$ is normal in $X$ if every order interval in $X$ is bounded in norm (see $[1,4]$ ).

Definition 1.1: A map $T: X \rightarrow X$ is said to be isotone increasing if for $x, y \in X$ and $x \leq y$ we have $T x \leq T y$.

The measure of noncompactness [2] of $A \subseteq X$ is defined by

$$
\alpha(A)=\inf \left\{r>0: A \subseteq \cup_{i=1}^{n} A_{i} \text { and } \operatorname{diam}\left(A_{i}\right) \leq r \text { for } i \in\{1, \ldots, n\}\right\} .
$$

Definition 1.2: Let $Q \subseteq X$. A map $T: Q \rightarrow X$ is said to be countably condensing if $T(Q)$ is bounded and if for any countably bounded set $A$ of $Q$ with $\alpha(A)>0$ we have $\alpha(T(A))<\alpha(A)$.

In Section 2 we prove new common fixed point theorems for a pair of single valued maps and in Section 3 we prove the multivalued analogue of these theorems. The results in this paper complement and extend results in the literature; see [3] and the references therein.

\section{Pairs of Single-Valued Mappings}

In this section we prove some common fixed point theorems for a pair of mappings defined on a closed subset of an ordered Banach space $X$.

Condition $D_{Q}$ : Let $Q \subseteq X$. Two maps $S, T: Q \rightarrow Q$ are said to satisfy condition $D_{Q}$ if for any countable set $A$ of $Q$ and for any fixed $a \in Q$ the condition

$$
A \subseteq\{a\} \cup S(A) \cup T(A)
$$

implies $\bar{A}$ is compact.

Definition 2.1: Two maps $S$ and $T$ on an ordered Banach space $X$ into itself are said to be weakly isotone increasing if $S x \leq T S x$ and $T x \leq S T x$ for all $x \in X$. Similarly $S$ and $T$ are said to be weakly isotone decreasing if $S x \geq T S x$ and $T x \geq$ $S T x$ for all $x \in X$. Also two mappings $S$ and $T$ are called weakly isotone if they are either weakly isotone increasing or weakly isotone decreasing.

Now we are ready to prove our main result.

Theorem 2.1: Let $B$ be a closed subset of an ordered Banach space $X$ and let $S, T: B \rightarrow B$ be two continuous and weakly isotone mappings satisfying condition $D_{B}$. Then $S$ and $T$ have a common fixed point.

Proof: Let $x \in B$ be arbitrary. Suppose $S$ and $T$ are weakly isotone increasing. Define a sequence $\left\{x_{n}\right\} \subseteq B$ as follows:

$$
x_{0}=x, x_{2 n+1}=S x_{2 n}, x_{2 n+2}=T x_{2 n+1} \text { for } n \geq 0 .
$$

Note $x_{1}=S x_{0} \leq T S x_{0}=T x_{1}=x_{2}$ and so we have

$$
x_{1} \leq x_{2} \leq x_{3} \leq \ldots \ldots .
$$

Let $A=\left\{x_{0}, x_{1}, \ldots ..\right\}$. Now $A$ is countable and

$$
A=\left\{x_{0}\right\} \cup\left\{x_{1}, x_{3}, \ldots .\right\} \cup\left\{x_{2}, x_{4}, \ldots .\right\} \subseteq\left\{x_{0}\right\} \cup S(A) \cup T(A) .
$$


Now $S$ and $T$ satisfy condition $D_{B}$ so $\bar{A}$ is compact. Thus $\left\{x_{n}\right\}$ has a convergent subsequence which converges to say $x^{\star} \in B$. However $\left\{x_{n}\right\}$ is nondecreasing from above, so the original sequence $\left\{x_{n}\right\}$ converges to $x^{\star} \in B$. Also the continuity of $T$ and $S$ imply

$$
x^{\star}=\lim _{n \rightarrow \infty} x_{2 n+1}=\lim _{n \rightarrow \infty} S x_{2 n}=S\left(\lim _{n \rightarrow \infty} x_{2 n}\right)=S\left(x^{\star}\right)
$$

and

$$
x^{\star}=\lim _{n \rightarrow \infty} x_{2 n+2}=\lim _{n \rightarrow \infty} T x_{2 n+1}=T\left(\lim _{n \rightarrow \infty} x_{2 n+1}\right)=T\left(x^{\star}\right) .
$$

Thus $S$ and $T$ have a common fixed point. The case when $S$ and $T$ are weakly isotone decreasing is similar.

Corollary 2.1: Let $B$ be a closed subset of an ordered Banach space $X$ and let $S, T: B \rightarrow B$ be two continuous, countably condensing and weakly isotone mappings. Then $S$ and $T$ have a common fixed point.

Proof: The result follows from Theorem 2.1 once we show $S$ and $T$ satisfy condition $D_{B}$. To see this let $A$ be a countable subset of $B, a \in B$ fixed, and $A \subseteq\{a\} \cup S(A) \cup T(A)$. Now $A$ is bounded since $S$ and $T$ are condensing (so in particular $S(B)$ and $T(B)$ are bounded). Now if $\alpha(A) \neq 0$ then

$$
\alpha(A) \leq \max \{\alpha(\{a\}), \alpha(S(A)), \alpha(T(A))\}<\alpha(A),
$$

which is a contradiction. Thus $\alpha(A)=0$, so $\bar{A}$ is compact.

In our next result let $X$ be a Banach space, $K$ a cone in $X$ and let $\bar{x}, \bar{y} \in X$ be such that $\bar{x} \leq \bar{y}$. Also $[\bar{x}, \bar{y}]$ denotes an order interval in $X$ as described in Section 1 .

Condition $R$ : Two weakly isotone maps $S, T:[\bar{x}, \bar{y}] \rightarrow[\bar{x}, \bar{y}]$ are said to satisfy condition $R$ if for any countable set $A$ of $[\bar{x}, \bar{y}]$,

(i) the condition

$$
A \subseteq\{\bar{x}\} \cup S(A) \cup T(A)
$$

implies $\bar{A}$ is compact if $S$ and $T$ are weakly isotone increasing,

(ii) whereas the condition

$$
A \subseteq\{\bar{y}\} \cup S(A) \cup T(A)
$$

implies $\bar{A}$ is compact if $S$ and $T$ are weakly isotone decreasing.

Corollary 2.2: Suppose $S, T:[\bar{x}, \bar{y}] \rightarrow[\bar{x}, \bar{y}]$ are two continuous and weakly isotone mappings satisfying condition $R$. Then $S$ and $T$ have a common fixed point.

Proof: If $S$ and $T$ are weakly isotone increasing we define a sequence $\left\{x_{n}\right\} \subseteq[\bar{x}, \bar{y}]$ as in (2.1) with $x_{0}=\bar{x}$, whereas if $S$ and $T$ are weakly isotone decreasing we define a sequence $\left\{x_{n}\right\} \subseteq[\bar{x}, \bar{y}]$ as in $(2.1)$ with $x_{0}=\bar{y}$.

Corollary 2.3: Suppose $S, T:[\bar{x}, \bar{y}] \rightarrow[\bar{x}, \bar{y}]$ are two continuous, countably condensing and weakly isotone mappings. Then $S$ and $T$ have a common fixed point $x^{\star} \in[\bar{x}, \bar{y}]$.

Remark 2.1: If in Corollary 2.3 the cone $K$ is normal in $X$ then the condition that $S([\bar{x}, \bar{y}])$ and $T([\bar{x}, \bar{y}])$ are bounded in the definition of countably condensing is automatically satisfied since $[\bar{x}, \bar{y}]$ is bounded in norm.

Remark 2.2: Assume $S, T:[\bar{x}, \bar{y}] \rightarrow[\bar{x}, \bar{y}]$ are two continuous weakly isotone mappings satisfying condition $R$ and in addition suppose $S$ and $T$ are isotone increasing. 
(i) Suppose $S$ and $T$ are weakly isotone increasing.

We know from Corollary 2.2 that the sequence $\left\{x_{n}\right\}$ defined by

$$
x_{0}=\bar{x}, x_{2 n+1}=S x_{2 n}, x_{2 n+2}=T x_{2 n+1} \text { for } n \geq 0
$$

converges to a common fixed point $x^{\star} \in[\bar{x}, \bar{y}]$ of $S$ and $T$.

Now let $z \in[\bar{x}, \bar{y}]$ be any common fixed point of $S$ and $T$. We claim that $x^{\star} \leq z$. To see this notice since $S$ and $T$ are isotone increasing that

$$
x_{1}=S \bar{x} \leq S z=z, x_{2}=T x_{1} \leq T z=z, \ldots \ldots .
$$

Thus $x_{n} \leq z$ for all $n \in\{1,2, \ldots\}$. Since $\left\{x_{n}\right\}$ converges to $x^{\star}$ we have $x^{\star} \leq z$.

(ii) Suppose $S$ and $T$ are weakly isotone decreasing.

We know from Corollary 2.2 that the sequence $\left\{x_{n}\right\}$ defined by

$$
x_{0}=\bar{y}, x_{2 n+1}=S x_{2 n}, x_{2 n+2}=T x_{2 n+1} \text { for } n \geq 0
$$

converges to a common fixed point $y^{\star} \in[\bar{x}, \bar{y}]$ of $S$ and $T$.

Now let $z \in[\bar{x}, \bar{y}]$ be any common fixed point of $S$ and $T$. Similar reasoning as in (i) guarantees that $z \leq y^{\star}$.

\section{Pairs of Multivalued Mappings.}

Let $X$ be an ordered Banach space and let $2^{X}$ (respectively $C(X)$ ) denote the family of all nonempty (respectively, nonempty closed) subsets of $X$. Let $A, B \in 2^{X}$. Then $A \leq B$ means $a \leq b$ for all $a \in A$ and $b \in B$.

Definition 3.1: A map $T: X \rightarrow 2^{X}$ is said to be isotone increasing if for $x$, $y \in X$ and $x \leq y, x \neq y$ we have $T x \leq T y$.

Definition 3.2: Two maps $S, T: X \rightarrow 2^{X}$ are said to be weakly isotone increasing if for any $x \in X$ we have $S x \leq T y$ for all $y \in S x$ and $T x \leq S y$ for all $y \in T x$. $S$ and $T$ are called weakly isotone decreasing if for any $x \in X$ we have $S x \geq T y$ for all $y \in S x$ and $T x \geq S y$ for all $y \in T x$. Also two mappings $S$ and $T$ are called weakly isotone if they are either weakly isotone increasing or weakly isotone decreasing.

Condition $D_{Q}$ : Let $Q \subseteq X$. Two maps $S, T: Q \rightarrow 2^{Q}$ are said to satisfy condition $D_{Q}$ if for any countable set $A$ of $Q$ and for any fixed $a \in Q$ the condition

$$
A \subseteq\{a\} \cup S(A) \cup T(A)
$$

implies $\bar{A}$ is compact; here $T(A)=\cup_{x \in A} T x$.

Theorem 3.1: Let $B$ be a closed subset of an ordered Banach space $X$ and let $S, T: B \rightarrow C(B)$ be two closed (i.e. have closed graph) weakly isotone mappings satisfying condition $D_{B}$. Then $S$ and $T$ have a common fixed point.

Proof: Let $x \in B$ be arbitrary. Suppose $S$ and $T$ are weakly isotone increasing. Define a sequence $\left\{x_{n}\right\} \subseteq B$ as follows:

$$
x_{0}=x, x_{2 n+1} \in S x_{2 n}, x_{2 n+2} \in T x_{2 n+1} \text { for } n \geq 0 .
$$


Note $x_{1} \in S x_{0}$ and since $S x_{0} \leq T y$ for all $y \in S x_{0}$ we have $S x_{0} \leq T x_{1}$. In particular $S x_{0} \leq x_{2}$, and so $x_{1} \leq x_{2}$. As a result we have

$$
x_{1} \leq x_{2} \leq x_{3} \leq \ldots \ldots \text {. }
$$

Let $A=\left\{x_{0}, x_{1}, \ldots ..\right\}$. Now $A$ is countable and

$$
A=\left\{x_{0}\right\} \cup\left\{x_{1}, x_{3}, \ldots .\right\} \cup\left\{x_{2}, x_{4}, \ldots .\right\} \subseteq\left\{x_{0}\right\} \cup S(A) \cup T(A) .
$$

Now $S$ and $T$ satisfy condition $D_{B}$ so $\bar{A}$ is compact. Thus $\left\{x_{n}\right\}$ has a convergent subsequence which converges to say $x^{\star} \in B$. However $\left\{x_{n}\right\}$ is nondecreasing from above, so the original sequence $\left\{x_{n}\right\}$ converges to $x^{\star} \in B$. Now

$$
x_{2 n} \rightarrow x^{\star}, x_{2 n+1} \rightarrow x^{\star}, x_{2 n+1} \in S x_{2 n},
$$

together with the fact that $S$ has closed graph implies $x^{\star} \in S x^{\star}$. Similarly $x^{\star} \in T x^{\star}$. The case when $S$ and $T$ are weakly isotone decreasing is similar.

Definition 3.3: Let $Q \subseteq X$. A map $T: Q \rightarrow 2^{X}$ is said to be countably condensing if $T(Q)$ is bounded and if for any countably bounded set $A$ of $Q$ with $\alpha(A)>0$ we have $\alpha(T(A))<\alpha(A)$.

Essentially the same reasoning as in Corollary 2.1 establishes the following result.

Corollary 3.1: Let $B$ be a closed subset of an ordered Banach space $X$ and let $S, T: B \rightarrow C(B)$ be two closed, countably condensing, weakly isotone mappings. Then $S$ and $T$ have a common fixed point.

In our next result let $X$ be a Banach space, $K$ a cone in $X$ and let $\bar{x}, \bar{y} \in X$ be such that $\bar{x} \leq \bar{y}$. Also $[\bar{x}, \bar{y}]$ denotes an order interval in $X$ as described in Section 1.

Condition $R$ : Two weakly isotone maps $S, T:[\bar{x}, \bar{y}] \rightarrow 2^{[\bar{x}, \bar{y}]}$ are said to satisfy condition $R$ if for any countable set $A$ of $[\bar{x}, \bar{y}]$,

(i) the condition

$$
A \subseteq\{\bar{x}\} \cup S(A) \cup T(A)
$$

implies $\bar{A}$ is compact if $S$ and $T$ are weakly isotone increasing,

(ii) whereas the condition

$$
A \subseteq\{\bar{y}\} \cup S(A) \cup T(A)
$$

implies $\bar{A}$ is compact if $S$ and $T$ are weakly isotone decreasing.

Corollary 3.2: Suppose $S, T:[\bar{x}, \bar{y}] \rightarrow C([\bar{x}, \bar{y}])$ are two closed, weakly isotone mappings satisfying condition $R$. Then $S$ and $T$ have a common fixed point.

Proof: If $S$ and $T$ are weakly isotone increasing we define a sequence $\left\{x_{n}\right\} \subseteq[\bar{x}, \bar{y}]$ as in (3.1) with $x_{0}=\bar{x}$, whereas if $S$ and $T$ are weakly isotone decreasing we define a sequence $\left\{x_{n}\right\} \subseteq[\bar{x}, \bar{y}]$ as in (3.1) with $x_{0}=\bar{y}$.

\section{References}

[1] Amann, H., Fixed point equations and nonlinear eigenvalue problems in Banach spaces, SIAM Review 18(1976), 620-709.

[2] Banas, J. and Goebel, K., Measures of Noncompactness in Banach Spaces, Marcel Dekker, New York 1980. 
[3] Dhage, B.C.,Common fixed point mappings on ordered Banach spaces with applications, Math. Sci. Research Journal 6(2002), 210-220.

[4] Guo, D. and Lakshmikantham, V., Nonlinear Problems in Abstract Cones, Academic Press, New York 1988. 


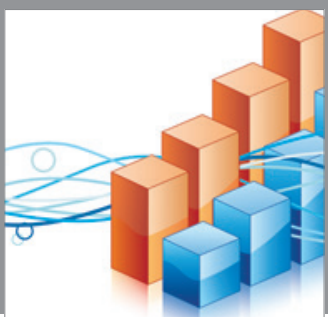

Advances in

Operations Research

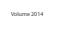

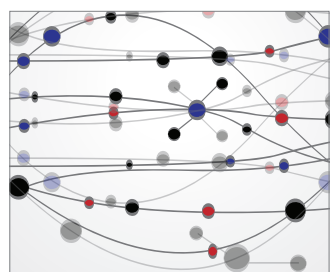

\section{The Scientific} World Journal
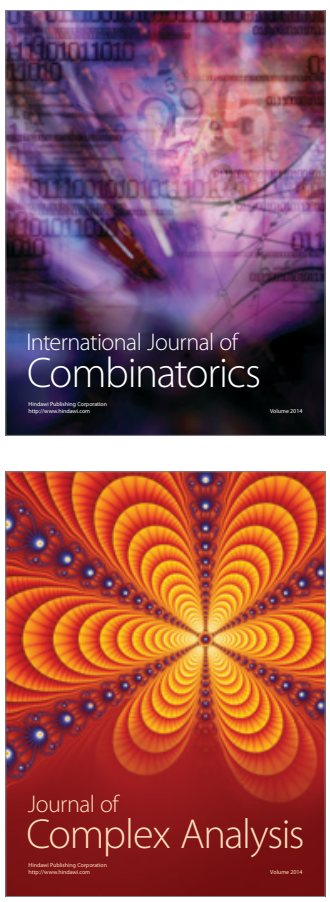

International Journal of

Mathematics and

Mathematical

Sciences
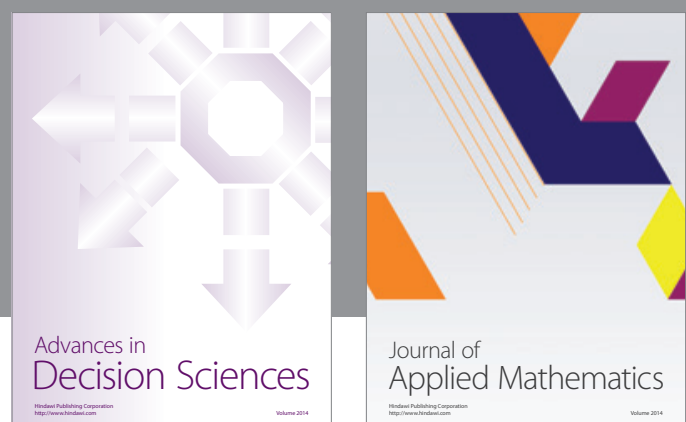

Journal of

Applied Mathematics
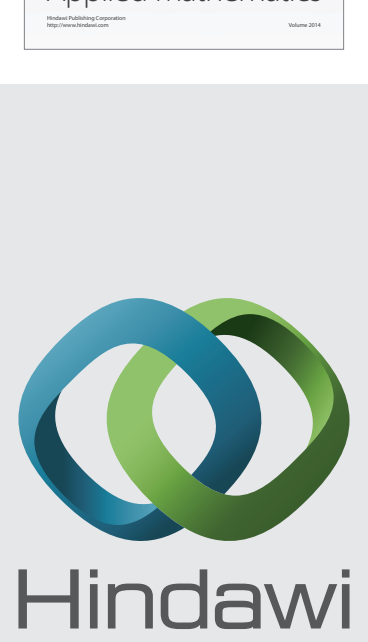

Submit your manuscripts at http://www.hindawi.com
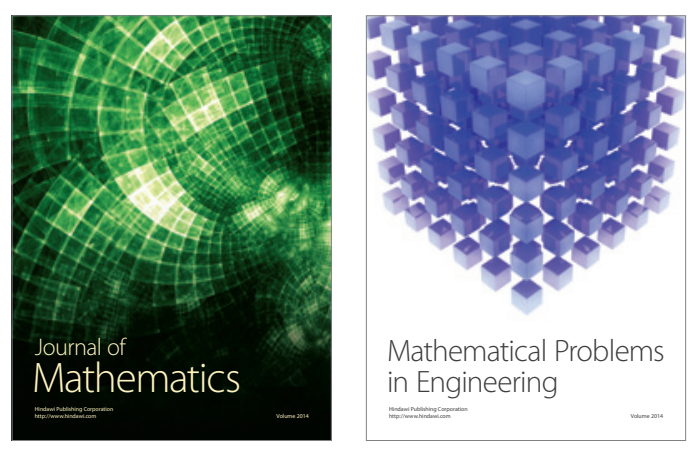

Mathematical Problems in Engineering
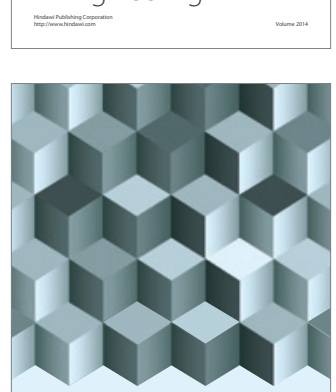

Journal of

Function Spaces
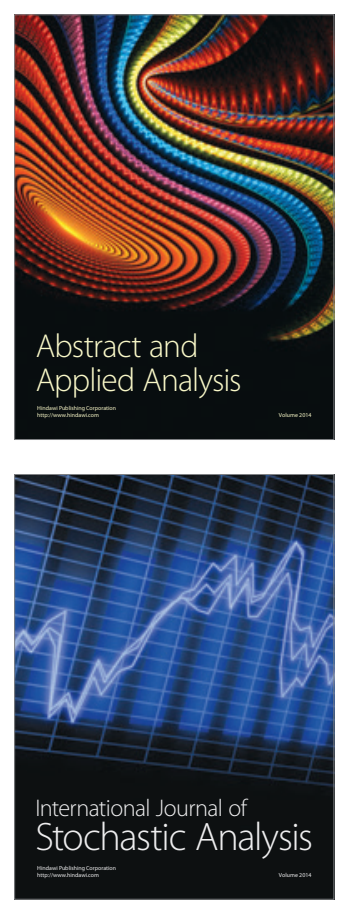

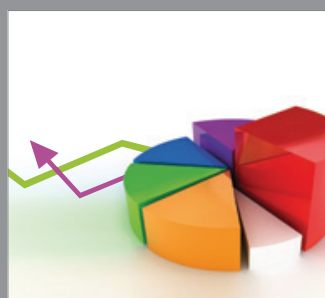

ournal of

Probability and Statistics

Promensencen
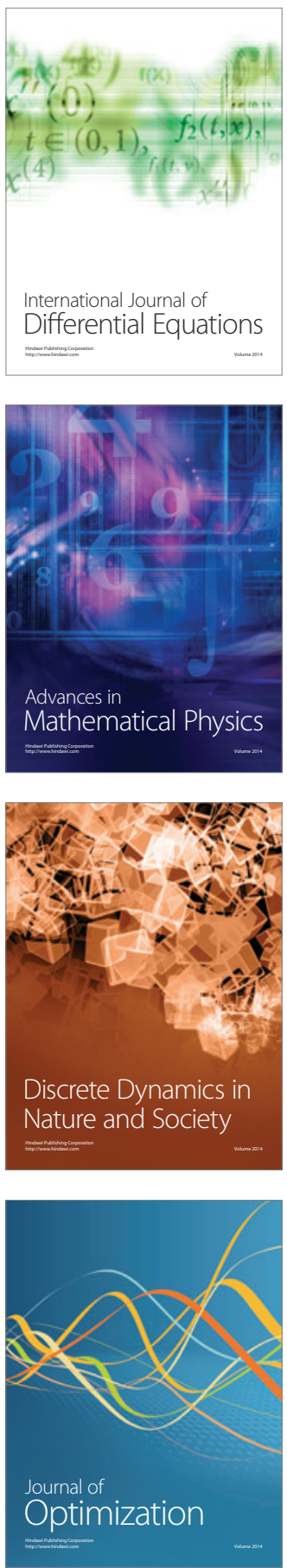